\title{
Beta secretase 1-dependent amyloid precursor protein processing promotes excessive vascular sprouting through NOTCH3 signalling
}

\author{
Claire S. Durrant $\mathbb{1}^{1,2,3}$, Karsten Ruscher ${ }^{4,5}$, Olivia Sheppard ${ }^{1}$, Michael P. Coleman ${ }^{1,2,6}$ and Ilknur Özen (1) ${ }^{1,5,7}$
}

\begin{abstract}
Amyloid beta peptides (AB) proteins play a key role in vascular pathology in Alzheimer's Disease (AD) including impairment of the blood-brain barrier and aberrant angiogenesis. Although previous work has demonstrated a proangiogenic role of $A \beta$, the exact mechanisms by which amyloid precursor protein (APP) processing and endothelial angiogenic signalling cascades interact in $A D$ remain a largely unsolved problem. Here, we report that increased endothelial sprouting in human-APP transgenic mouse (TgCRND8) tissue is dependent on $\beta$-secretase (BACE1) processing of APP. Higher levels of A $\beta$ processing in TgCRND8 tissue coincides with decreased NOTCH3/ JAG1 signalling, overproduction of endothelial filopodia and increased numbers of vascular pericytes. Using a novel in vitro approach to study sprouting angiogenesis in TgCRND8 organotypic brain slice cultures (OBSCs), we find that BACE1 inhibition normalises excessive endothelial filopodia formation and restores NOTCH3 signalling. These data present the first evidence for the potential of BACE1 inhibition as an effective therapeutic target for aberrant angiogenesis in $A D$.
\end{abstract}

\section{Introduction}

Alzheimer's disease (AD) is closely associated with alterations in the vascular system ${ }^{1}$. Multiple studies in humans and animal models have described pathological vascular changes in $\mathrm{AD}^{2}$, including disruption to the neurovascular unit ${ }^{3}$ and blood-brain barrier ${ }^{4}$, increased microvessel density ${ }^{3,5,6}$, arteriolar and venular tortuosity $^{7,8}$ and vascular $A \beta$ accumulation ${ }^{9}$. Such changes will likely compromise the effective delivery of oxygen and nutrients to the brain, so understanding whether vascular alterations are a cause or consequence of aspects of $\mathrm{AD}$

\footnotetext{
Correspondence: Michael P. Coleman (mc469@cam.ac.uk) or Ilknur Özen (ilknur.ozen@med.lu.se)

'John van Geest Centre for Brain Repair, Forvie Site, Robinson Way, Cambridge CB2 OPY, UK

${ }^{2}$ The Babraham Institute, Babraham Research Campus, Cambridge CB22 3AT, UK

Full list of author information is available at the end of the article These authors contributed equally: Michael P. Coleman, Ilknur Özen Edited by B. Joseph
}

pathology, notably $A \beta$ accumulation, is required in order to design effective therapies.

Amyloid beta peptides, particularly $A \beta_{1-42}$, are hallmarks of $\mathrm{AD}^{10}$. These peptides are the result of sequential proteolytic cleavage of amyloid precursor protein (APP) by $\beta$ - and $\gamma$-secretase enzyme activity. Whilst it is reported that synapse loss is the best correlate of clinical outcome in $\mathrm{AD}^{11}$, it is unclear as to whether pathological APP processing products drive this effect by a direct action on neurons, or indirectly such as through aberrant angiogenesis. Despite widespread interest in the role of brain vasculature in $\mathrm{AD}$, little is known about how amyloid-induced vascular changes alter pathological sprouting angiogenesis.

Sprouting angiogenesis is responsible for the formation of new blood vessels in the cortex. This process (both pathological and physiological) encompasses sequential events including; sprouting at the vascular front of endothelial cells, extension of sprouts, the formation of new vascular loops and pericyte recruitment ${ }^{12-14}$. Pericyte 
recruitment is closely linked to endothelial cell sprouting. Endothelial tip cells secrete platelet derived growth factor (PDGF) that activates platelet derived growth factor receptor beta (PDGFR $\beta$ ) on pericytes to induce their migration to the sprout ${ }^{15,16}$. Endothelial sprouting is regulated by the NOTCH signalling pathway ${ }^{17}$. NOTCH receptors (NOTCH1-4) undergo proteolytic processing via $\gamma$-secretase in a manner comparable to that of $\mathrm{APP}^{18,19}$ resulting in the hypothesis that interactions between these signalling pathways could underlie the angiogenic pathology in $\mathrm{AD}^{20,21}$.

Whilst indirect measures of angiogenic activity, such as vessel density, can be studied in post mortem brains, active processes, such as filopodia extension from tip cells, are notoriously difficult to observe in vivo. Mechanistic exploration of pathological angiogenesis using drugs, such as BACE1, is also complicated by issues of blood-brain barrier penetration and peripheral effects (such as metabolic changes) that may confound interpretation of results $^{22}$. To permit mechanistic exploration of the relationship between amyloid pathology, $\mathrm{NOTCH}$ signalling and pathological angiogenesis, we used organotypic brain slice cultures (OBSCs), which provide an excellent experimental platform for such studies. OBSCs initially retain a dense network of capillaries and neurovascular units alongside maintenance of neuronal architecture and non-neuronal cell populations ${ }^{23-25}$. Crucially, OBSCs provide a three-dimensional culture system that supports the formation of new blood vessels and are amenable to pharmacological manipulation, live imaging and repeated measurements without interference from peripheral systems $^{26-29}$. We and others have previously shown that OBSCs are powerful tools for investigating the progression of AD-like alterations including $\mathrm{A} \beta$ accumulation ${ }^{30}$, synaptic disruption $^{27,31}$ and cerebrovascular damage ${ }^{32}$.

In this study, we find evidence for early pathological angiogenesis in the brains of postnatal TgCRND8 mice which was recapitulated in OBSCs. In addition to increased vessel density, OBSCs from TgCRND8 mice showed an increase in sprouting angiogenesis, that could be completely blocked by BACE1 inhibition. We investigate the mechanisms by which pathological APP processing and $\mathrm{NOTCH}$ signalling interact to induce excessive vascular sprouting and discuss the implications for the blood vessel pathology seen in AD.

\section{Results}

TgCRND8 mice show vascular abnormalities in the cortex

Vascular abnormalities have been widely reported in a range of amyloidosis mouse models, with conflicting reports demonstrating both increased and decreased microvessel density in adult transgenic mice ${ }^{3,5,33}$. Here, we sought to examine whether TgCRND8 mice show evidence of early pathological angiogenesis, as a potential reaction to the initiating steps of amyloidosis, where therapeutic intervention is more likely to be effective. We studied P7 TgCRND8 mouse post mortem cortex to determine whether a mutant huAPP transgene (and associated dysregulated amyloid processing), leads to pathological changes in the organisation of the vascular network and endothelial cell physiology during postnatal development $(30,31)$ (Fig. 1). PECAM-1 (a marker of endothelial cells) expressing cortical blood vessels of P7 TgCRND8 mice (Fig. 1b, b ") appeared to be more tortuous than cortical vessels of P7 WT mice (Fig. 1a, $\mathrm{a}^{\prime}$ ). When assessing PDGFR $\beta^{+}$ pericyte coverage of blood vessels in P7 TgCRND8 cortex, we found an $\sim 2$-fold higher pericyte coverage when compared to WT (Fig. 1c) with coverage becoming almost complete (Fig. 1b). We also found a significant increase in PECAM- $1^{+}$vessel density (Fig. $1 \mathrm{~d})$, with no significant alteration in vessel diameter (Fig. 1e).

\section{Characterisation of sprouting angiogenesis in organotypic brain slice cultures}

We next sought to establish potential mechanisms by which the presence of a mutant huAPP transgene results in pathological angiogenesis. For this we required an experimental system in which key angiogenic processes could be easily observed and manipulated. We established an ex vivo cortical organotypic brain slice culture (OBSC) system to analyse this highly regulated process and found we were able to identify multiple stages of sprouting angiogenesis in OBSCs from wild-type (WT) mice (summarised in Fig. 2e) that were not observable in post mortem brain (Fig. 1). PECAM-1 staining showed a dense network of blood vessels expressing basal membrane protein laminin in 7-days in vitro cortical slices (Fig. 2a). Endothelial tip cells were found either along the trunk of PECAM- $1^{+}$blood vessels with few filopodia (Fig. 2b, asterisk) or at the leading edge of vascular sprouts extending numerous filopodia (Fig. 2b, c) indicating active sprouting angiogenesis. The filopodia of some vascular sprouts were also found to engage with those of a nearby endothelial tip cells to form a bridge and the formation of new blood vessels (Fig. 2d).

PDGFR $\beta^{+}$pericytes were found around the OBSC blood vessels and with long cytoplasmic processes surrounding the abluminal surface of endothelium (Fig. 3a-c). High magnification confocal imaging showed that PDGFR $\beta$ expressing pericytes were closely associated with the PECAM- $1^{+}$vascular sprouts (Fig. 3d, e, framed image) and astrocytes within neurovascular units (Fig. $3 \mathrm{f}-\mathrm{h}$ ) at 7 days in vitro. Taken together, this demonstrates the utility of OBSCs as a tool to assess different steps of sprouting angiogenesis, including filopodia formation and pericyte coverage. 


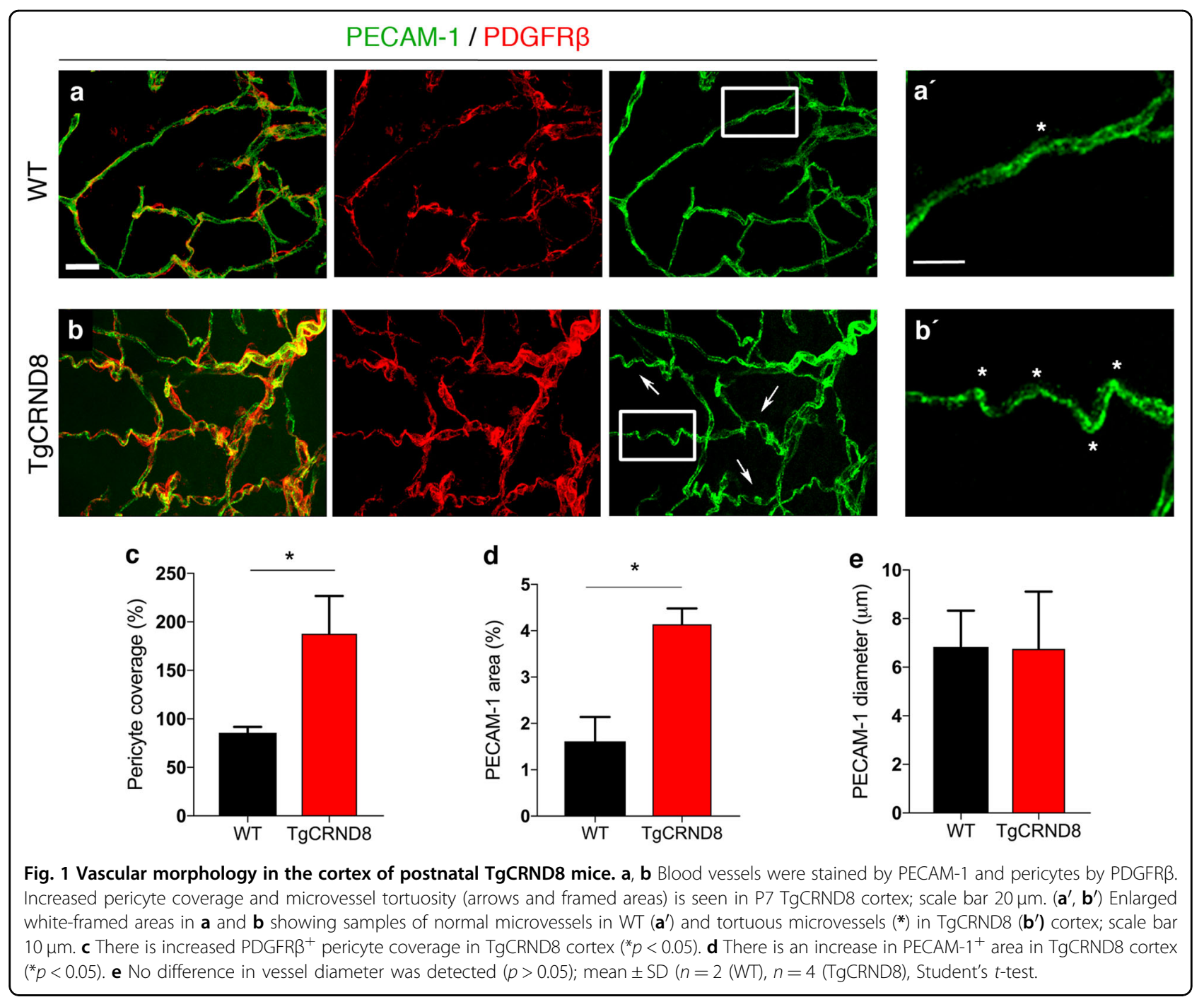

Excessive vascular sprouting is associated with increased vascular density and number of pericytes in TgCRND8 OBSCs

To determine whether OBSCs recapitulated our in vivo observations, we next looked for signs of vascular abnormalities in 7 days in vitro TgCRND8 OBSCs (Fig. 4). The capillary density (as measured by area of PECAM-1 staining) was significantly higher in TgCRND8 OBSCs compared to cultures from WT littermates (Fig. 4a, b). PECAM- ${ }^{+}$cells were found to co-localise with Ki67 (a marker of cell division) (Supplementary Fig. 1), potentially indicating increased endothelial proliferation as a contributor to increased vascular density. Confocal microscopy also revealed an increase in the number of filopodia found both at the leading edge, as well as along the body, of the vascular sprouts in TgCRND8 cultures (Fig. 4c) alongside a $50 \%$ increase in the number of filopodia at the vascular sprout of the blood vessels (Fig. 4d). Interestingly, OBSCs from an alternative amyloidosis mouse model
(5xFAD) showed similar changes, with a significant increase in PECAM-1 ${ }^{+}$vessel length and area (Supplementary Fig. $2 \mathrm{a}-\mathrm{c}$ ) and a substantial increase in filopodia number (Supplementary Fig. 2d, e) compared to WT littermate controls, indicative of conserved mechanisms between models.

In 7 days in vitro TgCRND8 OBSCs, the number of PDGFR $\beta^{+}$pericytes around the capillaries was significantly higher than WT controls (Fig. 5a, b) even when normalised to the increased density of blood vessels (Fig. 5c). The increased number of PDGFR $\beta^{+}$pericytes was correlated with an upregulation of PDGFR $\beta$ protein expression as measured by western blot (Fig. 5d, e).

\section{Inhibition of BACE1 activity normalises vascular density} and hypersprouting in TgCRND8 OBSCs

To investigate if higher vascular density and filopodia number depend on the increased production of $A \beta$ seen in TgCRND8 OBSCs, we applied the BACE1 


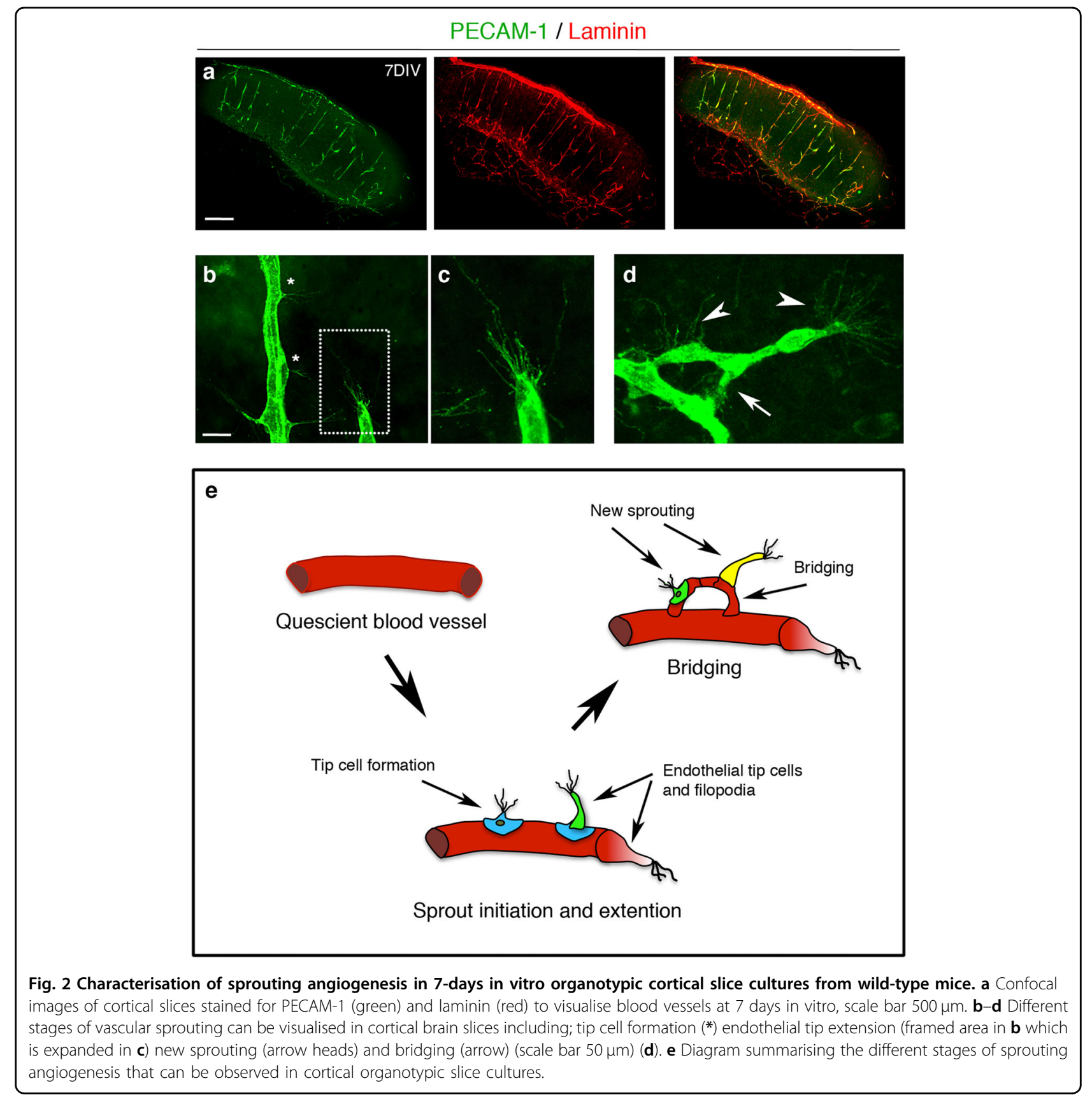

inhibitor LY2886721 to OBSCs for 7 days in vitro (Fig. 6a). This inhibitor has been extensively characterised both in vivo and in vitro and found to be a potent and highly selective BACE inhibitor (with essentially no inhibition of cathepsin $\mathrm{D}$, pepsin, renin or other similar aspartyl proteases) $)^{34}$. Using ELISA, we found that BACE1 inhibitor completely abolished the generation of $A \beta_{1-40}$ and $A \beta_{1-42}$ in the TgCRND8 OBSC culture medium (Fig. 6b) in agreement with previous characterisation of this inhibitor $^{34}$. Interestingly, $\mathrm{A} \beta$ was found to co-localise with blood vessels in TgCRND8 OBSCs, but was absent in WT vessels (Supplementary Fig. 3a, b). Vascular $A \beta$ was completely abolished by BACE1 inhibitor treatment (Supplementary Fig. 3b). BACE1 inhibition reduced the vascular density in TgCRND8 slices back to WT levels, with no additional effect on WT cultures (Fig. 6c-e). Quantification of PECAM-1 $1^{+}$capillaries revealed a twofold increase in total vessel length in TgCRND8 OBSCs, which was restored to WT levels after BACE1 inhibition (Fig. 6f). Similarly, BACE1 inhibition reduced the excessive sprouting activity of PECAM-1 ${ }^{+}$endothelial cells in TgCRND8 OBSCs, significantly lowering the number of 


\section{PDGFRß / PECAM-1 / DAPI}
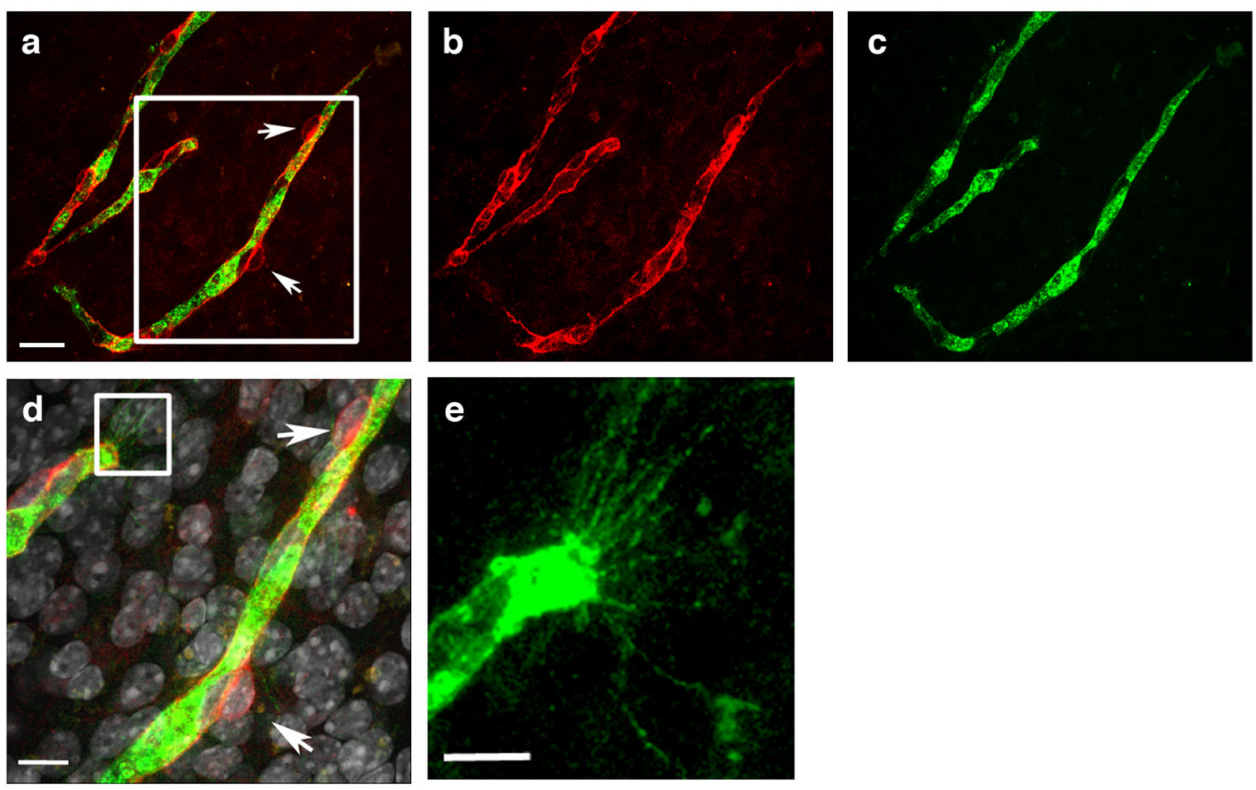

PDGFRß / PECAM-1 / GFAP / DAPI
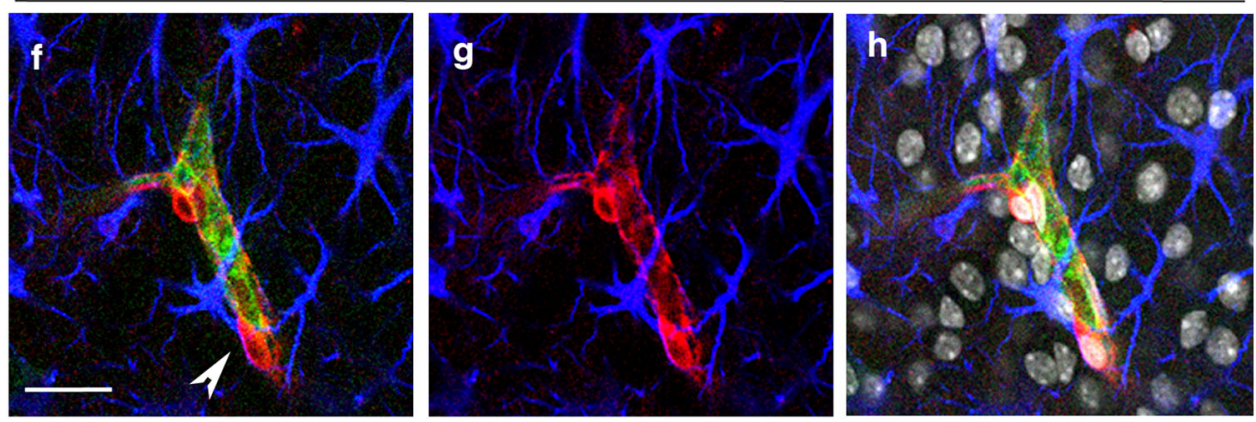

Fig. 3 Pericytes are well preserved in organotypic cortical slices at 7 days in vitro. a-c Confocal images showing PECAM-1 ${ }^{+}$blood vessels (green) $(\mathbf{a}, \mathbf{c})$ covered by PDGFRß ${ }^{+}$pericytes (red, arrows $(\mathbf{a}, \mathbf{b})$ ) in 7 days in vitro WT cortical slices; scale bar $20 \mu$ m. $\mathbf{d}$ Higher magnification images of the framed area in a with DAPI counterstain depicting nuclei (grey). Pericytes (arrows) can be seen on the PECAM- $1^{+}$angiogenic blood vessels; scale bar $10 \mu \mathrm{m}$. e depicts enlarged area framed in $\mathbf{d}$ showing clear vascular sprouting. $\mathbf{f}-\mathbf{h}$ Confocal z-stacks show a preserved cytoarchitecture, consisting of PECAM- $1^{+}$blood vessels (green), surrounded by PDGFRß ${ }^{+}$pericytes (red) and GFAP ${ }^{+}$astrocytes (blue) (DAPI = grey). Arrow marks the close association between the pericytes and astrocytes; scale bar $20 \mu \mathrm{m}$.

filopodia at the leading edge of the vascular sprout back to WT levels (Fig. 6g-i). Conversely, direct application of synthetic huA $\beta_{1-42}$ to WT cultures resulted in a significant increase in PECAM- $1^{+}$vessel density and length (Supplementary Fig. 4a-d), further supporting a hypothesis whereby the overproduction of $A \beta$ in TgCRND8 tissue can trigger pathological angiogenesis.

Despite significant rescue of hypersprouting (Fig. 6) we found that BACE1 inhibition did not normalise the increased levels of PDGFR $\beta$ in TgCRND8 OBSCs (Supplementary Fig. 5). Indeed, BACE1 inhibition increases the levels of PDGFR $\beta$ in WT OBSCs, but this is uncoupled from the lack of change seen in
PECAM-1 ${ }^{+}$vessel coverage, length and filopodia number (Fig. 6).

\section{BACE1 inhibition restores Notch3 and Jag1 mRNA levels in TgCRND8 cortical slices}

Given that modulating APP/A $\beta$ metabolism via BACE1 inhibition resulted in normalisation of hypersprouting, we hypothesised that interaction between $\mathrm{A} \beta$ peptide processing and $\mathrm{NOTCH}$ signalling might explain the endothelial hypersprouting observed in TgCRND8 mice. To test this hypothesis, we examined the mRNA levels of key components of the NOTCH signalling pathway, NOTCH1, NOTCH3, JAG1, JAG2 and DLL4, in control 

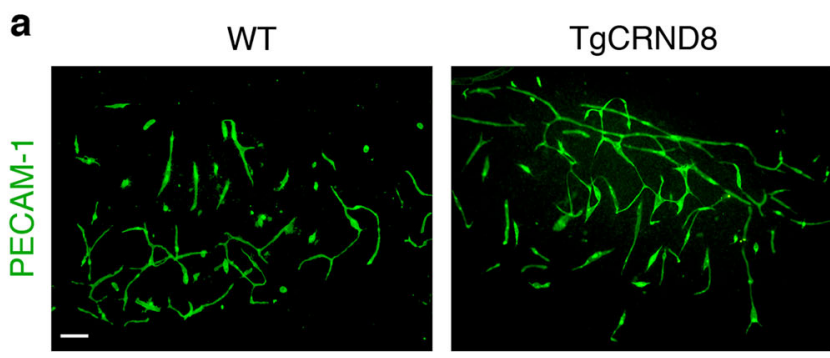

b

C
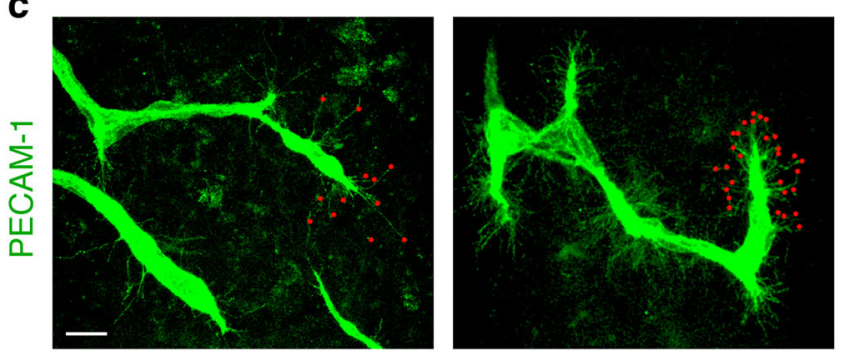

d
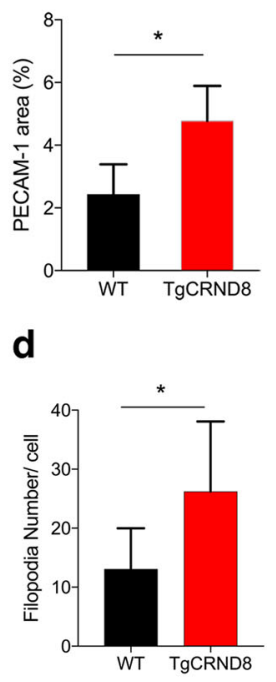

Fig. 4 TgCRND8 organotypic cortical slices show increased vascular density and excessive filopodia formation when compared to wildtype cultures. a Representative confocal images showing blood vessel density (PECAM-1, green) in 7 days in vitro WT and TgCRND8 slices; scale bar $100 \mu \mathrm{m}$. b Quantification of PECAM- $1^{+}$area (as percentage of the total image) reveals a significantly higher blood vessel density in TgCRND8 cortical slices vs. WT slices. (mean \pm SD $\left(n=5\right.$ (WT), $n=4$ (TgCRND8), ${ }^{*} P<0.05$ Student's $t$-test). c Confocal images showing endothelial cells extending numerous finger-like filopodia at the forefront of vascular sprouts in 7 days in vitro WT and TgCRND8 cortical slices, visualised by PECAM-1 labelling. Red dots highlight vascular sprouting tips, scale bar $20 \mu \mathrm{m}$. d Quantification shows that the number of filopodia per cell is significantly higher in TgCRND8 when compared to WT slices. (mean \pm SD, $(n=14$ (WT), $n=13$ (TgCRND8), * $P<0.05$ Student's $t$-test).

vs. BACE-inhibitor treated TgCRND8 and WT littermate OBSCs. Real-time quantitative PCR analysis showed that mRNA levels of Notch3 (Fig. 7a) and Jag1 (Fig. 7b) were significantly lower in TgCRND8 OBSCs when compared to the WT controls, whilst expression of Notch1, Jag2 and DLL4 were not significantly changed (Fig. 7c-e). In all, $5 \mu \mathrm{M}$ BACEI inhibitor treatment for 7 days in vitro normalised both Notch3 and Jag1 mRNA expression back to the levels observed in WT cultures (Fig. 7a, b). We found no significant changes in the mRNA expression of Notch1, Jag2, DLL4 in TgCRND8 or WT slices after BACE1 inhibitor treatment (Fig. 7c-e). Interestingly, application of synthetic A $\beta$ to WT slices for 3 days in vitro resulted in a reduction in Notch3 mRNA (Supplementary Fig. 4e) but did not alter the levels of Jag1 mRNA (Supplementary Fig. 4f), potentially indicating that changes to Notch3 are upstream to alterations in Jag1.

\section{BACE1 inhibition induces cleavage of NOTCH3 intracellular domain (NICD3) in TgCRND8 cortical slices}

Finally, we analysed how higher production of $A \beta$ affects NOTCH3 and NOTCH1 signalling activity in TgCRND8 OBSCs (Fig. 7f-i). Translocation of the $\mathrm{NOTCH}$ intracellular domain (NICD) into the nucleus is a negative regulator of endothelial sprouting ${ }^{17}$, so we tested whether the reduction in Notch3 mRNA led to lower levels of NICD3. Western blot analysis showed a trend for reduced levels of NOTCH3 intracellular domain (NICD3) in TgCRND8 cortical slices (Fig. 7f, g). In contrast, BACE1 inhibitor treatment significantly increased NICD3 levels in TgCRND8 slices to at least the level of WT cortical cultures (Fig. 7f, g). Consistent with the mRNA levels of Notch1, there was no effect of genotype or BACE1 inhibition on the appearance of NOTCH1 intracellular domain (NICD1) (Fig. 7h, i).

\section{Discussion}

In this study, we explored the relationship between $A \beta$ processing, NOTCH signalling and pathological angiogenesis in cortical tissue from huAPP transgenic mice. We report that BACE1 inhibition can reverse the increased pathological angiogenesis observed in TgCRND8 OBSCs alongside restoration of $\mathrm{NOTCH}$ signalling. We find that pathological angiogenesis is an early event in TgCRND8 mice, and is detectable before amyloid plaque formation, synapse loss or cognitive deficits ${ }^{35}$. Restoration of physiological levels of angiogenesis early in the disease course may therefore be an important target for $\mathrm{AD}$ therapeutics.

This study took advantage of the OBSC system to explore mechanisms of pathological angiogenesis that are difficult to study in vivo. OBSCs are a potent, underused tool for exploring vascular phenotypes ${ }^{23,36,37}$ with few prior studies seeking to explore this in combination with $\mathrm{AD}$ models ${ }^{32,38}$. Interestingly, the increased sprouting 


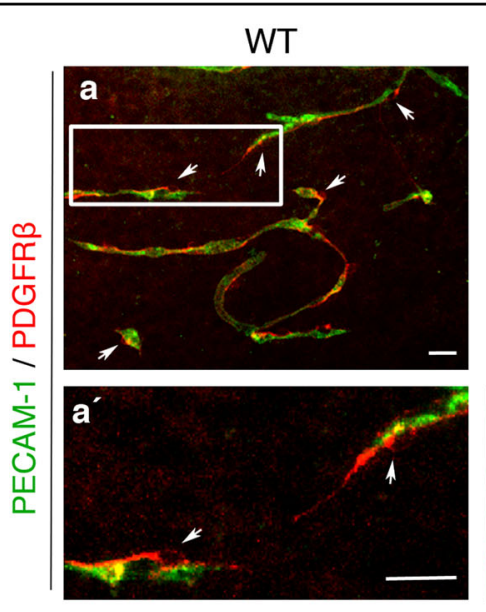

d

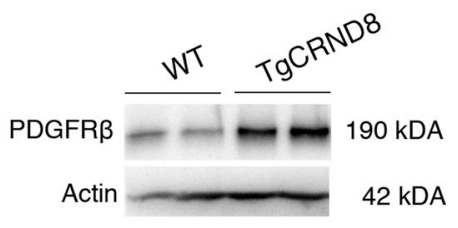

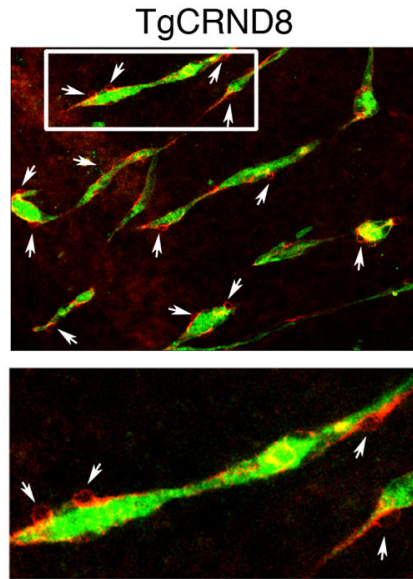

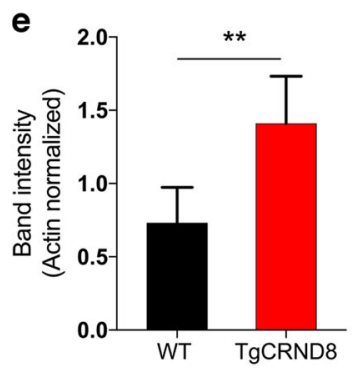

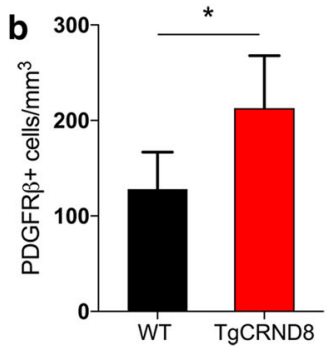

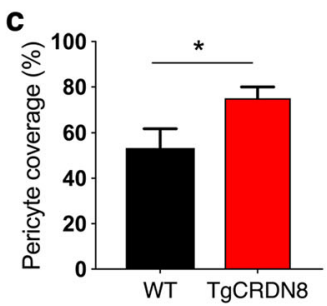

Fig. 5 Increased pericyte number and PDGFR $\beta$ expression in TgCRND8 organotypic cortical slices. a Representative confocal images showing PDGFR $\beta^{+}$pericytes (arrows) around blood vessels (PECAM-1, green) in 7 days in vitro WT and TgCRND8 slices; scale bar $50 \mu \mathrm{m}$. PDGFR $\beta^{+}$pericytes are closely associated with cortical microvessels. $\mathbf{a}^{\prime}$ The framed areas in $\mathbf{a}$ are enlarged, scale bar $50 \mu \mathrm{m}$. $\mathbf{b}$ Quantification of PDGFR $\beta^{+}$pericytes reveals an increased number in 7 days in vitro TgCRND8 slices when compared to WT (data are expressed as cell numbers per mm ${ }^{3}$. c Quantification of PDGFR ${ }^{+}$staining area normalised to PECAM- ${ }^{+}$staining area shows a significant increase in pericyte coverage in TgCRND8 OBSCs; mean \pm SD. $(n$ $=4(\mathrm{WT}), n=5$ (TgCRND8), ${ }^{*} P<0.05$ Student's $t$-test). Representative western blots (d) and quantification of PDGFR $\beta$ band intensity (e) in 7 days in vitro WT and TgCRND8 cortical slices, shows increased PDGFR $\beta$ in TgCRND8 cultures when normalised to Actin control $(n=5$ (WT), $n=4$ (TgCRND8) ${ }^{* *} P<0.01$ Student's $t$-test).

angiogenesis we observed in TgCRND8 OBSCs is more pronounced than we find in vivo at a similar age. The OBSC method likely stresses the tissue via the slicing injury and/or the isolation from the systemic vasculature; stimulating sprouting angiogenesis. By allowing visualisation of such processes in both WT and TgCRND8 tissue, the OBSC system appears to unmask a mechanism that underlies the vascular changes in $\mathrm{AD}$ models that may otherwise be overlooked.

Our finding that tissue from postnatal TgCRND8 mice shows pathological angiogenesis is of interest in the context of aging being a major risk factor for AD. Studies examining human brain microvessel density in the context of "normal" ageing have revealed inconsistent results (increased/decreased/no change) in aged vs. young individuals depending on brain region and microvessel type studied $^{39-42}$. Despite widespread interest in vascular pathology in $\mathrm{AD}$, there is also debate as to the relative roles of pro- and anti- angiogenic processes during disease progression ${ }^{1}$. For example, whilst some studies demonstrate increased angiogenesis in post mortem human AD or huAPP-mouse brain ${ }^{3,5,6,43}$, others report endothelial cell apoptosis and loss of vasculature ${ }^{33,44}$. It seems likely that such studies represent different stages of disease, with initial increases in angiogenesis (potentially as a result of rising $A \beta$ that occurs in ageing ${ }^{45,46}$ ) being overtaken by cell death in the end stages ${ }^{1}$. Clearly, more work is required to fully understand the complex relationships between age, $\mathrm{AD}$ and vascular pathology, although our work indicates pathological angiogenesis can be induced by dysregulated $A \beta$ processing even in the absence of ageing.

A potential role for $A \beta$ as a regulator of angiogenesis has been previously proposed from observations in both physiological and pathological conditions. In post mortem human AD brains, it has been shown that increased vascular density in the hippocampus ${ }^{6}$ and pericyte-mediated capillary restriction ${ }^{47}$ correlates with $A \beta$ load. In amyloid mouse models, immunisation with $A \beta$ peptides cleared plaques and restored capillary density to normal levels ${ }^{48}$ whilst inhibition of $A \beta$ aggregation reduced arteriolar $A \beta$ accumulation and tortuosity ${ }^{7}$. Application of synthetic $A \beta$ 


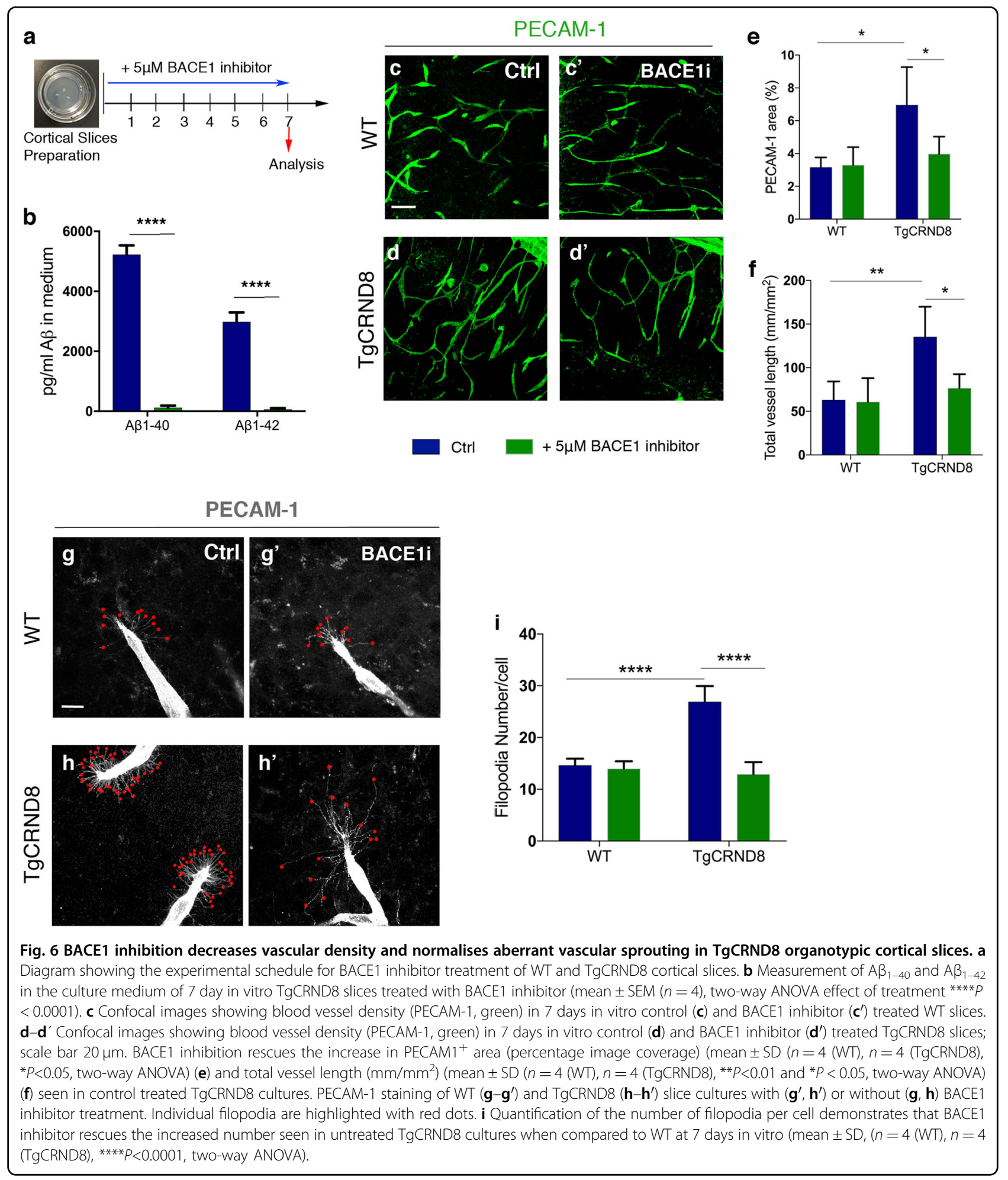

to a number of models has also highlighted its proangiogenic role with increased endothelial cell proliferation, capillary bed density and vascular sprouting seen both in vitro and in vivo ${ }^{20,49,50}$. Our use of a novel OBSC platform that endogenously expresses mutant APP with all its processing products allows for careful exploration of the effects of $A \beta$ as well as other APP-derived products on vascular pathology ${ }^{51-53}$. Our findings add weight to growing evidence that dysregulation of amyloid processing, or exogenously applied huA $\beta_{1-42}$ (Supplementary 


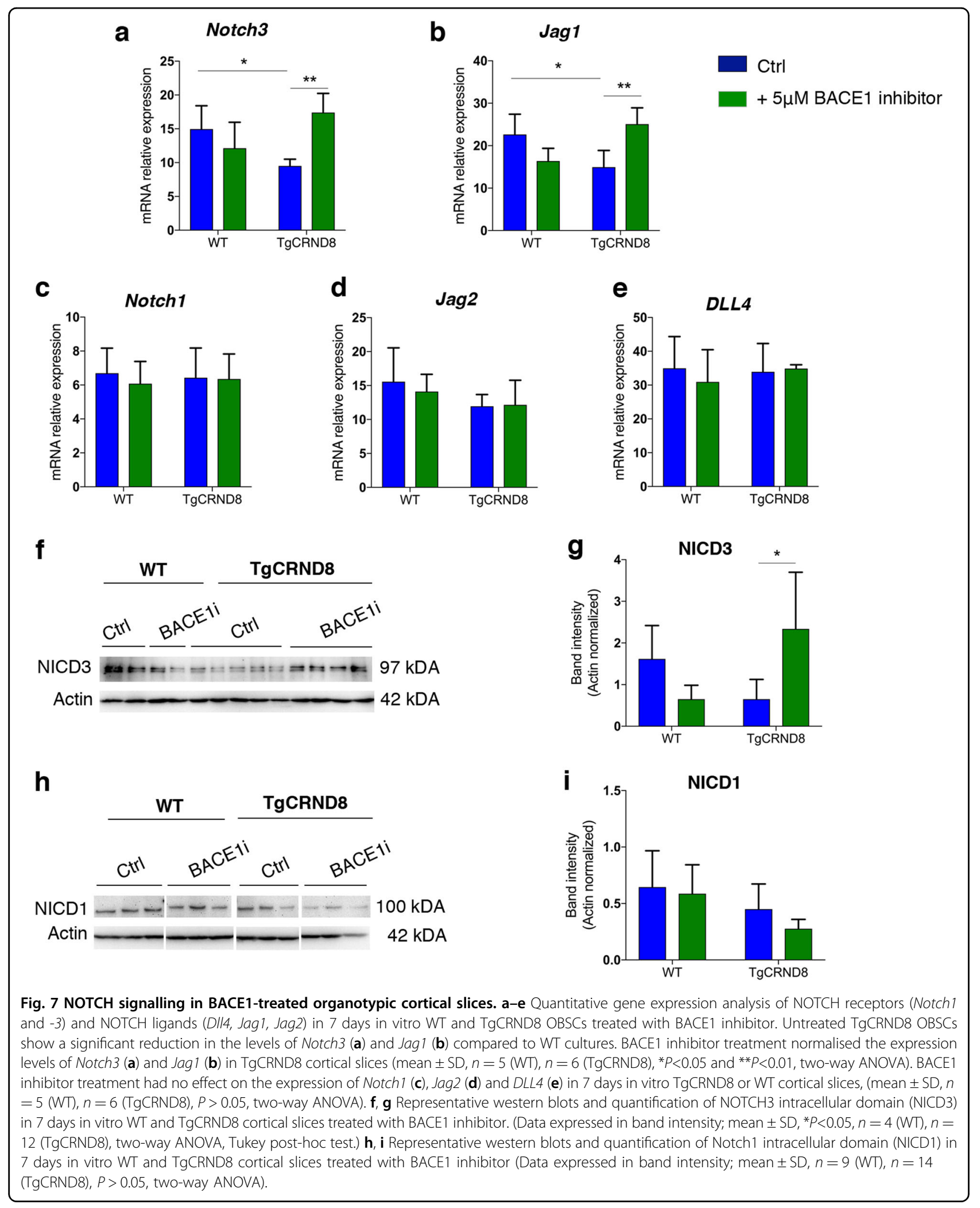


a

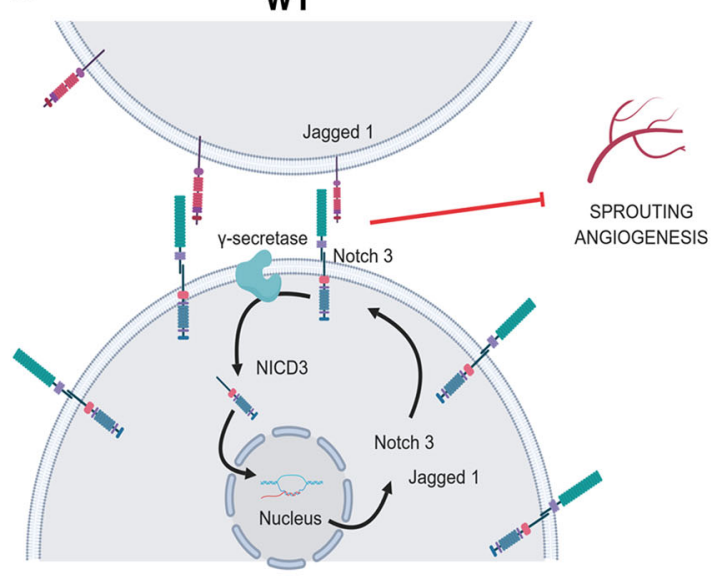

b

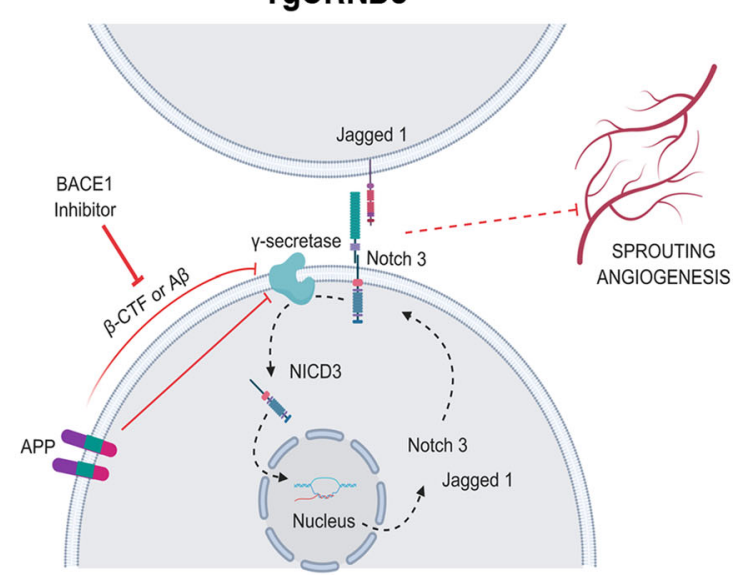

Fig. 8 Proposed mechanism for the enhancement of sprouting angiogenesis by BACE1-dependent APP processing. Schematic diagram of our working hypothesis for increased sprouting angiogenesis in TgCRND8 (b) compared to WT (a) tissue. Increased APP processing by BACE1 in TgCRND8 OBSCs competes with NOTCH3 for $\gamma$-secretase or reduces $\gamma$-secretase activity, thereby lowering transcriptional signalling through NICD. This reduces Notch3-Jag1 expression via autoregulatory mechanisms, thereby releasing the inhibitory influence on sprouting angiogenesis. Created with BioRender.

Fig. 4), can stimulate pathological angiogenesis. It is worth noting, that whilst we were unable to analyse the exact form (monomeric, oligomeric etc.) of $A \beta$ present in the TgCRND8 cultures (due to the presence of serum in the culture medium and very low concentration in the slice tissue itself) we know from our previous work that the $A \beta$ present, and thus likely responsible for our observed phenotypes, is soluble, thioflavin-S negative and is not sequestered in extracellular plaques ${ }^{27}$.

A key finding in this study is that overproduction of $A \beta$ in TgCRND8 OBSCs is accompanied by reduced expression of the angiogenesis suppressor NOTCH3 and its ligand JAG1, providing a novel mechanistic insight into how amyloid pathology potentially impacts angiogenesis in AD. Previous studies have shown that Notch3 knockout increases retinal vascular density and endothelial tip formation ${ }^{54}$ and silencing NOTCH3 in tumours promotes pathological angiogenesis $^{55}$. NOTCH ligand JAG1 has also been implicated in angiogenic processes, with Jag1 targeting antisense oligonucleotides potentiating FGF-responsive tube formation and invasion in vitro ${ }^{56}$. There are multiple potential mechanisms by which Notch3 and Jag1 could be downregulated in postnatal TgCRND8 tissue, which we summarise in our working hypothesis (Fig. 8).

$\mathrm{NOTCH}$ proteins and NOTCH ligands are substrates for the $\gamma$-secretase presenilin ${ }^{57}$, resulting in the production of NICD which translocates to the nucleus to regulate gene expression (Fig. 8a). Cleavage of NOTCH3 by $\gamma$-secretase has been found to induce Notch3 and Jag1 transcription via autoregulatory mechanisms ${ }^{58}$. Previous work has also shown that NOTCH3 activation (by cleavage to NICD3) is prevented by treatment with $\gamma$-secretase inhibitors ${ }^{59}$ which results in increased angiogenic sprouting ${ }^{60}$. Interestingly, this effect is mimicked by the application of synthetic monomeric $A \beta$ potentially pointing to an enzymatic feedback inhibition, whereby high levels of $A \beta$ lower the activity of $\gamma$-secretase ${ }^{49}$. This study aligns with our findings that application of synthetic A $\beta$ to WT OBSCs results in increased microvessel density alongside a reduction in Notch3 mRNA (Supplementary Fig. 4). In TgCRND8 tissue (Fig. 8b) increased levels of $A \beta$ may act via this mechanism to inhibit the efficacy of $\gamma$-secretase, reducing levels of NOTCH3 cleavage and so lowering Notch3 and Jag1 transcription, ultimately resulting in increased sprouting angiogenesis. Alternatively, other APP processing products may also have inhibitory effects on $\gamma$-secretase. $\beta$-CTF, the result of BACE1 cleavage of APP, contains a region $\left(A \beta_{17-23}\right)$ that has been found to modulate $\gamma$-secretase activity by non-competitive inhibition ${ }^{61}$ and a similar role has been proposed for the APP intracellular domain $(\mathrm{AID})^{19}$. Alternatively, increased expression of APP, or enhanced processing of APP through $\gamma$-secretase may directly compete with $\mathrm{NOTCH}$ ligands for enzymatic availability $^{62}$.

In this study, we find that excessive endothelial filopodia formation and reduced NOTCH3/JAG1 signalling in TgCRND8 OBSCs can be normalised via application of BACE1 inhibitor (Fig. 8b) indicating a likely role for BACE1-dependent APP processing products. Interestingly, PDGFR $\beta$ protein remained elevated in BACE1-inhibitor treated TgCRND8 cultures and such treatment increased PDGFR $\beta$ in WT cultures (Supplementary Fig. 5), without impacting vascular phenotypes (Fig. 6). The role of pericytes in angiogenesis is dynamic and at times opposing ${ }^{63}$, with 
contrasting studies demonstrating their presence promotes early angiogenesis and vessel survival ${ }^{13,64-66}$, whilst inhibiting endothelial cell proliferation or inducing vessel regression in later stages ${ }^{67-69}$. This raises the possibility that increased pericyte expression could be a compensatory attempt at terminating pathological angiogenesis in TgCRND8 cultures which only becomes effective after normalisation of $\mathrm{A} \beta$ levels. BACE1 treatment in WT cultures may end ongoing physiological angiogenesis, resulting in the proliferation of pericytes on the stabilised, mature vessels. Alternatively, BACE1 may have an independent role in regulating PDGFR $\beta$ signalling that is uncoupled, at least in the short-term, from alterations in endothelial filopodia or microvessel density. BACE1 inhibitors have previously been shown to inhibit angiogenesis and tumour growth ${ }^{70}$. BACE1 knockout mice show reduced vascular density ${ }^{71}$ and treatment of zebrafish with BACE1 inhibitors was found to induce similar deficits, with application of $A \beta$ restoring normal vascularisation ${ }^{72}$. Whilst we hypothesise that the normalisation of TgCRND8 angiogenesis after BACE1 inhibition is due to reduced levels of $A \beta$, we have to consider the possibility that BACE inhibition has a direct effect on angiogenic processes unrelated to APP. Other studies have found that BACE1 directly regulates JAG1 shedding, with $\mathrm{BACE} 1^{-1-}$ mice showing an increase in Jag1 levels and downstream NOTCH signalling ${ }^{73}$. This seems an unlikely mechanism in our system, however, due to the observation that BACE1 inhibition has no effect on the levels of Jag1 (Fig. 7b) or vascular density (Fig. 6) in WT OBSCs and that application of synthetic A $\beta$ alone increases PECAM- $1^{+}$ vessel density (Supplementary Fig. 4). From our results, however, it is possible that BACE1 plays an independent role in pericyte recruitment/PDGFR $\beta$ expression, which warrants further exploration. Future studies could also examine whether the rescue effect of BACE1 inhibition on pathological angiogenesis is reversible, providing greater insight into the dynamics of angiogenesis in response to changes in amyloid processing.

Further mechanistic studies are needed to better understand the interplay between NOTCH signalling and APP processing mechanisms in AD. Here, we propose that APP overexpression or feedback inhibition from high $A \beta / \beta-C T F$ concentration in TgCRND8 OBSCs reduces $\gamma$-secretasedependent cleavage of NOTCH3 (Fig. 8). Our results also indicate that there may be a safe level of BACE1 inhibition that can restore physiological levels of angiogenesis, without inducing angiogenic deficits in healthy tissue.

\section{Methods \\ Mice}

TgCRND8 mice ${ }^{35}$ were maintained on a mixed BL6:sv129 background. TgCRND8 mice overexpress human APP with both the Swedish (K670N/M671L) and Indiana (V717F) mutations. $5 \mathrm{xFAD}$ mice $^{74}$ were maintained on a C57BL/6 $\mathrm{x}$
SJL background. 5xFAD mice express human APP with the Swedish (K670N/M671L), Florida (I716V) and London (V717I) mutations, alongside human PSEN1 with the M146L and L286V mutations. TgCRND8 or 5xFAD heterozygote males were bred with wild-type background matched females to produce both wild-type and transgenic heterozygote littermates. Animals were kept on a $12 \mathrm{~h}: 12 \mathrm{~h}$ light:dark cycle at a constant temperature of $19^{\circ} \mathrm{C}$ in a pathogen-free environment. All animal work was approved by the Babraham Institute Animal Welfare and Ethical Review Body, the University of Cambridge and the UK Home Office, and carried out in accordance with the Animals (Scientific Procedures) Act, 1986.

\section{Organotypic brain slice cultures}

Cortical organotypic brain slice cultures were made from humanely killed P6-P9 littermates of either sex according to the method of de Simoni et al and our previous work ${ }^{27,31,75}$. Briefly, after schedule 1, brains were kept on ice in dissection buffer (EBSS supplemented with $25 \mathrm{mM}$ HEPES and $1 \mathrm{x}$ Penicillin/Streptomycin). In all, 350- $\mu$ m-thick sagittal sections of cortex were cut using a Leica VT1000S vibratome and slices collected using a modified sterile $3 \mathrm{ml}$ Pasteur pipette. On average 6 cortical slices were collected per pup and stored in dissection buffer on ice until plating. For longterm culture, slices were transferred, in a class II hood, onto sterile Millicell ${ }^{\circledast}$ membrane inserts (Millipore PICM0RG50) in $35 \mathrm{~mm}$ culture dishes (Nunc). 3 cortical slices from the same pup were plated to a single membrane, with two dishes made per animal. Inserts were maintained in $1 \mathrm{ml}$ of maintenance medium according to the following recipe: $50 \%$ MEM with Glutamax-1 (Life Technologies 42360-024), 25\% Heat-inactivated horse serum (Life Technologies: 26050-070), 23\% EBSS (Life Technologies: 24010-043), 0.65\% D-Glucose (Sigma:G8270), 2\% Penicillin/Streptomycin (Life Technologies 15140-122) and 6 units/ml Nystatin (Sigma N1638). Membrane inserts were handled with sterile forceps and the medium was changed $100 \% 4 \mathrm{~h}$ after plating and at 4 div. OBSCs were maintained at $37^{\circ} \mathrm{C}, 5 \% \mathrm{CO}_{2}$ and high humidity for $7 \mathrm{div}$. For BACE1 inhibition experiments, 1 culture per pup was treated with $5 \mu \mathrm{M}$ BACE1 inhibitor LY2886721 (Selleckchem S2156) (previously reported to have high potency and specificity both in vivo and in vitro ${ }^{34}$ ) and compared with a DMSO treated control from the same animal. Cultures were treated for the entire 7 days in vitro. Synthetic huA $\beta_{1-42}$ was prepared according to a previously published protocol $^{76}$ (see supplementary methods).

\section{Immunohistochemistry \\ OBSCS}

OBSCs were fixed for $20 \mathrm{~min}$ in 4\% PFA in phosphate buffered saline (PBS), washed three times in PBS, then blocked for $1 \mathrm{~h}$ in PBS supplemented with $0.5 \%$ Triton X100 and 3\% normal Goat Serum (Sigma G9023). Slices 
were incubated at $4{ }^{\circ} \mathrm{C}$ in primary antibody (diluted in blocking solution) overnight. In order to detect PDGFR $\beta$, heat-mediated antigen retrieval was performed in $10 \mathrm{mM}$ citrate buffer ( $\mathrm{pH} 6.0$ ) for $40 \mathrm{~min}$ at $80^{\circ} \mathrm{C}$ prior to primary antibody incubation. Slices were washed a further three times in $0.5 \%$ Triton-X100 in PBS (PBS-TX) then incubated with secondary antibodies (Life Technologies and Jackson) (1:500 dilution in blocking solution for $2 \mathrm{~h}$ at $4{ }^{\circ} \mathrm{C}$ ). Three final PBS-TX washes were conducted before slices were mounted on slides and images captured using a Leica Confocal Microscope. Primary antibodies used: rabbit anti-PDGFR $\beta$ (28E1) (1:200, Cell Signalling, Cat. No: 3169S), rat anti-PECAM-1 (1:400, BD, Cat. No: 550274), rabbit anti-laminin (1:200, Sigma, Cat. No: L9393), Mouse MOAB-2 (pan A $)$ ) (1:1000, Merck-Millipore, Cat. No: MABN254) Rabbit Ki67 (1:1000, Abcam, Cat. No: ab15580) secondary staining was conducted using species-specific fluorophore-conjugated (Streptavidin Alexa 488, Molecular Probes; Cy3 or Cy5, Jackson,) or biotin-conjugated secondary antibodies (Jackson). DAPI $(1 \mu \mathrm{g} / \mathrm{mL}$, Sigma) was used to counterstain nuclei.

\section{Cryosections of postnatal mouse brain}

P7 WT and TgCRND8 pups, of either sex were culled via cervical dislocation followed by decapitation. Brains were removed, snap-frozen, and embedded in Tissue$\mathrm{Tek}^{\circledR}$ OCT compound (Sakura Finetek, The Netherlands). Cryosections were cut at $20 \mu \mathrm{m}$ thickness and fixed in either ice cold acetone or 2\% PFA for $20 \mathrm{~min}$ and airdried. Cryosections were then stained for PECAM-1 and PDGFR $\beta$ as described above.

\section{Microscopy and image analysis}

Images were captured via an epifluorescence microscopy system (Leica DM6000B) or using confocal microscopes (Leica, Zeiss LSM780). Figures were composed using Photoshop CS5 software. All analysis was done blind to genotype and/or treatment condition.

\section{Quantification of pericyte number and coverage}

To quantify the number of PDGFR $\beta$-positive pericytes, cells were counted using NIH Image J Cell Counter tool. A maximum projection of fifteen-micrometre z-stacks was acquired from cortex slices freshly derived from WT and TgCRND8. Three pictures were taken at 40x for each slice. The areas of PDGFR $\beta^{+}$pericytes and PECAM- $1^{+}$blood vessels were separately subjected to threshold processing and the respective signals for each image was calculated using NIH Image J Area Measurement tool. Pericyte coverage was determined as a percentage (\%) of PDGFR $\beta^{+}$pericyte area covering PECAM $-1^{+}$capillary surface area per field (ROI) $733 \times 733 \mu \mathrm{m}$. Three slices per animal were analysed.

\section{Capillary density, length and filopodia quantification}

For PECAM-1 ${ }^{+}$capillary area, sections were analysed with Leica confocal microscope. Three pictures were taken at 20x for each section. ROI size of $733 \times 733 \mu \mathrm{m}$ for confocal images were utilised. The area covered by PECAM $-1^{+}$capillaries was analysed using the NIH ImageJ area measurement tool where pictures were subjected to threshold processing to produce a binary image. The area of PECAM-1 coverage was expressed as percentage of the total area, three slices per WT and TgCRND8 pups $(n=$ 4-5 for each genotype) were analysed. The filopodia of vascular sprouts were analysed using z-stacked PECAM$1^{+}$blood vessels.

\section{Protein extraction and western Blot}

OBSCs were scraped off the membrane insert using a scalpel and transferred to $2 x$ Laemelli buffer supplemented with $10 \% 2$-mercaptethanol $(50 \mu \mathrm{L}$ per three slices). Samples were boiled for $10 \mathrm{~min}$ then frozen at $-20{ }^{\circ} \mathrm{C}$ until use. Ten micrograms of protein were separated on a $10 \%$ SDS polyacrylamide gel then transferred onto PVDF membranes. Membranes underwent blocking $(20 \mathrm{mM}$ Tris, $136 \mathrm{mM} \mathrm{NaCl}, \mathrm{pH}$ 7.6, $0.1 \%$ Tween 20 and 5\% nonfat dry milk) before incubation with primary antibody anti-NOTCH1/ NICD1 (1:750, Abcam, Cat No: ab3294), NOTCH3/ NICD3 (1:1000, Abcam, Cat No: ab23426), PDGFR $\beta$ (28E1), (1:1000, Cell Signaling, Cat No: 3169 S), overnight at $4{ }^{\circ} \mathrm{C}$. overnight at $4{ }^{\circ} \mathrm{C}$. Signals were obtained by binding of a secondary anti-rabbit horse radish peroxidase (HRP) linked antibody (1:15000, Sigma-Aldrich, Cat No: A0545) and visualised by exposing the membrane to a charge-coupled device camera (LAS1000, Fujifilm, Tokyo, Japan) using a chemiluminescence kit (Merck Millipore, Billerica, MA, USA). Membranes were stripped and reprobed for $\beta$-actin (diluted 1:50 000, Sigma-Aldrich, Cat No: A3854). After densitometric analysis using Image $J$ software, protein levels were calculated as percentage of $\beta$-actin expression.

\section{A $\beta$ ELISA}

Culture medium from 7 div OBSCs was assayed for human $A \beta_{1-40}$ and $A \beta_{1-42}$ using commercially available ELISA kits (Life Technologies: KHB3441 and KHB3481). Briefly, medium was incubated with $A \beta$ detection antibody for $3 \mathrm{~h}$, washed, and then incubated with an HRPconjugated antibody for $30 \mathrm{~min}$. After another wash step, stabilised chromogen was added for $30 \mathrm{~min}$ before the adding an acid-based stop solution. Absorbance was read at $450 \mathrm{~nm}$ using a PheraStar ELISA plate reader with the standard curve calculated using a 4-parameter fit. Concentration of $A \beta$ in the medium is expressed as $\mathrm{pg} / \mathrm{ml}$. Levels of $A \beta$ were compared between BACE1-treated and DMSO treated TgCRND8 cultures. 


\section{Quantification of gene expression by qPCR}

Slice cultures were scraped off the membrane and RNA extracted using an RNeasy mini kit (Qiagen). Briefly, three slices were homogenised in $350 \mu \mathrm{L}$ lysis buffer RLT supplemented with 1\% 2-mercaptethanol. In all, $350 \mu \mathrm{L} \mathrm{70 \%}$ ethanol (in nuclease free water) was then added and samples transferred to RNeasy RNA collection columns. After several wash steps described in the kit protocol, the RNA was eluted in $20 \mu \mathrm{L}$ nuclease free water, measured and quality tested using a Nanodrop ${ }^{\circledR}$ and frozen at $-80^{\circ} \mathrm{C}$ until use. cDNA synthesis was performed using Script ${ }^{\mathrm{TM}}$ CDNA Synthesis Kit (Bio-Rad). cDNA was analysed using real-time PCR SsoAdvanced ${ }^{\mathrm{TM}} \mathrm{SYBR}^{\circledR}$ Green Supermix from Bio-Rad and run on a Bio-Rad CFX96 real-time quantitative PCR (qPCR) system. Gene expression was normalised to the housekeeping gene GAPDH. Melt curve analyses were performed to ensure the specificity of qPCR product. Primer sequences are given in Supplementary Table 1 . Values are presented as mean \pm SD of three independent experiments, and within each experiment, triplicate samples were assessed.

\section{Statistical analysis}

Experimental sample sizes were selected via power analysis of preliminary data using an online calculator: http://powerandsamplesize.com. All samples from the same animal, under the same treatment conditions were averaged to produce a single biological replicate. Each experiment was performed on 2-3 independent occasions. Statistical analysis was conducted using Graph Pad Prism. Data are expressed as mean \pm SD. Two group comparison was performed by using Student's $t$-test (twosided) and multiple group comparison by one-way or twoway ANOVA followed by Tukey post-hoc test (to correct for multiple comparisons). For Western blot, after normalisation to the actin signal, the expression of each protein was compared using a two-way ANOVA, followed by Tukey post hoc test. Significance was set at $p<0.05$.

\section{Acknowledgements \\ This work was funded by Alzheimer's Research UK project grant ARUK- PG2015-24, the John and Lucille Van Geest Foundation, Swedish Brain Fund (FO2019-0254; FO2018-0316), the Hans-Gabriel and Alice Trolle-Wachtmeister Foundation and Sparbanksstiftelsen Färs \& Frosta. We would like to thank Helois Radford for their assistance in providing 5xFAD mouse pups.}

\section{Author details}

${ }^{1} J o h n$ van Geest Centre for Brain Repair, Forvie Site, Robinson Way, Cambridge CB2 OPY, UK. ${ }^{2}$ The Babraham Institute, Babraham Research Campus, Cambridge CB22 3AT, UK. ${ }^{3}$ Centre for Discovery Brain Sciences, University of Edinburgh, 1 George Square, Edinburgh EH8 9JZ, UK. ${ }^{4}$ Laboratory for Experimental Brain Research, Department of Clinical Sciences, Lund University, SE, Lund, Sweden. ${ }^{5}$ Lund Brain Injury Laboratory for Neurosurgical Research, Department of Clinical Sciences, Lund University, Lund, Sweden. ${ }^{6}$ Present address: John van Geest Centre for Brain Repair, Forvie Site, Robinson Way, Cambridge CB2 OPY, UK. ${ }^{7}$ Present address: Lund Brain Injury Laboratory for Neurosurgical Research, Wallenberg Neuroscience Center, Lund University, BMC A13, 22184 Lund, SwedenFundingOpen access funding provided by Lund University.

\section{Data availability}

The datasets generated and analysed in this study are available from the corresponding author on request.

Conflict of interest

The authors declare that they have no conflict of interest.

\section{Publisher's note}

Springer Nature remains neutral with regard to jurisdictional claims in published maps and institutional affiliations.

Supplementary Information accompanies this paper at (https://doi.org/ 10.1038/s41419-020-2288-4).

Received: 3 June 2019 Revised: 21 January 2020 Accepted: 22 January 2020 Published online: 06 February 2020

\section{References}

1. Govindpani, K. et al. Vascular dysfunction in alzheimer's disease: a prelude to the pathological process or a consequence of it? J. Clin. Med. 8, 651 (2019).

2. Jefferies, W. A. et al. Adjusting the compass: new insights into the role of angiogenesis in Alzheimer's disease. Alzheimers Res. Ther. 5, 64 (2013).

3. Giuliani, A. et al. Age-related changes of the neurovascular unit in the cerebral cortex of alzheimer disease mouse models: a neuroanatomical and molecular study. J. Neuropathol. Exp. Neurol. 78, 101-112 (2019).

4. Yamazaki, Y. et al. Selective loss of cortical endothelial tight junction proteins during Alzheimer's disease progression. Brain J. Neurol. https://doi.org/10.1093/ brain/awz011 (2019).

5. Biron, K. E., Dickstein, D. L., Gopaul, R. \& Jefferies, W. A. Amyloid triggers extensive cerebral angiogenesis causing blood brain barrier permeability and hypervascularity in Alzheimer's disease. PloS ONE 6, e23789 (2011).

6. Desai, B. S., Schneider, J. A., Li, J.-L., Carvey, P. M. \& Hendey, B. Evidence of angiogenic vessels in Alzheimer's disease. J. Neural Transm. 116, 587-597 (2009).

7. Dorr, A. et al. Amyloid- $\beta$-dependent compromise of microvascular structure and function in a model of Alzheimer's disease. Brain 135, 3039-3050 (2012).

8. Lai, A. Y. et al. Venular degeneration leads to vascular dysfunction in a transgenic model of Alzheimer's disease. Brain 138, 1046-1058 (2015).

9. Meyer, E. P., Ulmann-Schuler, A., Staufenbiel, M. \& Krucker, T. Altered morphology and $3 \mathrm{D}$ architecture of brain vasculature in a mouse model for Alzheimer's disease. Proc. Natl Acad. Sci. USA 105, 3587-3592 (2008).

10. Hardy, J. The amyloid hypothesis of Alzheimer's disease: progress and problems on the road to therapeutics. Science 297, 353-356 (2002).

11. Terry, R. D. et al. Physical basis of cognitive alterations in Alzheimer's disease: synapse loss is the major correlate of cognitive impairment. Ann. Neurol. 30, 572-580 (1991).

12. Chung, A. S. \& Ferrara, N. Developmental and pathological angiogenesis. Annu. Rev. Cell Dev. Biol. 27, 563-584 (2011).

13. Ozerdem, U. \& Stallcup, W. B. Early contribution of pericytes to angiogenic sprouting and tube formation. Angiogenesis 6, 241-249 (2003).

14. Potente, M., Gerhardt, H. \& Carmeliet, P. Basic and therapeutic aspects of angiogenesis. Cell 146, 873-887 (2011).

15. Hellström, M., Kalén, M., Lindahl, P., Abramsson, A. \& Betsholtz, C. Role of PDGF$B$ and PDGFR-beta in recruitment of vascular smooth muscle cells and pericytes during embryonic blood vessel formation in the mouse. Development 126, 3047-3055 (1999).

16. Lindblom, P. et al. Endothelial PDGF-B retention is required for proper investment of pericytes in the microvessel wall. Genes Dev. 17, 1835-1840 (2003).

17. Phng, L.-K. \& Gerhardt, H. Angiogenesis: a team effort coordinated by notch. Dev. Cell 16, 196-208 (2009).

18. Hartmann, D., Tournoy, J., Saftig, P., Annaert, W. \& De Strooper, B. Implication of APP secretases in notch signaling. J. Mol. Neurosci. 17, 171-181 (2001).

19. Roncarati, R. et al. The gamma-secretase-generated intracellular domain of beta-amyloid precursor protein binds Numb and inhibits Notch signaling. Proc. Natl Acad. Sci. USA 99, 7102-7107 (2002). 
20. Boscolo, E. et al. $\beta$ amyloid angiogenic activity in vitro and in vivo. Int. J. Mol. Med. 19, 581-587 (2007)

21. Ethell, D. W. An amyloid-notch hypothesis for Alzheimer's disease. Neuroscientist 16, 614-617 (2010).

22. Lahiri, D. K., Maloney, B., Long, J. M. \& Greig, N. H. Lessons from a BACE inhibitor trial: off-site but not off base. Alzheimers Dement. 10, S411-S419 (2014).

23. Hutter-Schmid, B., Kniewallner, K. M. \& Humpel, C. Organotypic brain slice cultures as a model to study angiogenesis of brain vessels. Front. Cell Dev. Biol. 3, 52 (2015).

24. Moser, K. V., Schmidt-Kastner, R., Hinterhuber, H. \& Humpel, C. Brain capillaries and cholinergic neurons persist in organotypic brain slices in the absence of blood flow. Eur. J. Neurosci. 18, 85-94 (2003).

25. Moser, K. V., Reindl, M., Blasig, I. \& Humpel, C. Brain capillary endothelial cells proliferate in response to NGF, express NGF receptors and secrete NGF after inflammation. Brain Res. 1017, 53-60 (2004).

26. Croft, C. L. \& Noble, W. Preparation of organotypic brain slice cultures for the study of Alzheimer's disease. F1000Research 7, 592 (2018).

27. Harwell, C. S. \& Coleman, M. P. Synaptophysin depletion and intraneuronal A $\beta$ in organotypic hippocampal slice cultures from huAPP transgenic mice. Mol. Neurodegener. 11, 44 (2016).

28. Holopainen, I. E. Organotypic hippocampal slice cultures: a model system to study basic cellular and molecular mechanisms of neuronal cell death, neuroprotection, and synaptic plasticity. Neurochem. Res. 30 1521-1528 (2005).

29. Humpel, C. Organotypic vibrosections from whole brain adult Alzheimer mice (overexpressing amyloid-precursor-protein with the Swedish-Dutch-lowa mutations) as a model to study clearance of beta-amyloid plaques. Front Aging Neurosci. 7, 47 (2015).

30. Novotny, R. et al. Conversion of synthetic $A \beta$ to in vivo active seeds and amyloid plaque formation in a hippocampal slice culture model. J. Neurosci. 36, 5084-5093 (2016).

31. Sheppard, O., Coleman, M. P. \& Durrant, C. S. Lipopolysaccharide-induced neuroinflammation induces presynaptic disruption through a direct action on brain tissue involving microglia-derived interleukin 1 beta. J. Neuroinflammation 16, 106 (2019).

32. Kniewallner, K. M., Foidl, B. M. \& Humpel, C. Platelets isolated from an Alzheimer mouse damage healthy cortical vessels and cause inflammation in an organotypic ex vivo brain slice model. Sci. Rep. 8, 15483 (2018).

33. Religa, P. et al. VEGF significantly restores impaired memory behavior in Alzheimer's mice by improvement of vascular survival. Sci. Rep. 3, 2053 (2013).

34. May, P. C. et al. The potent BACE1 inhibitor LY2886721 elicits robust central A $\beta$ pharmacodynamic responses in mice, dogs, and humans. J. Neurosci. 35 1199-1210 (2015).

35. Chishti, M. A. et al. Early-onset amyloid deposition and cognitive deficits in transgenic mice expressing a double mutant form of amyloid precursor protein 695. J. Biol. Chem. 276, 21562-21570 (2001).

36. Chip, S., Zhu, X. \& Kapfhammer, J. P. The analysis of neurovascular remodeling in entorhino-hippocampal organotypic slice cultures. J. Vis. Exp. https://doi.org/ 10.3791/52023 (2014).

37. Ullrich, C. \& Humpel, C. The pro-apoptotic substance thapsigargin selectively stimulates re-growth of brain capillaries. Curr. Neurovasc. Res. 6, 171-180 (2009).

38. Daschil, N. et al. L-type calcium channel blockers and substance P induce angiogenesis of cortical vessels associated with beta-amyloid plaques in an Alzheimer mouse model. Neurobiol. Aging 36, 1333 (2015).

39. Sonntag, W. E., Eckman, D. M., Ingraham, J. \& Riddle, D. R. in Brain Aging: Models, Methods, and Mechanisms (ed. Riddle, D. R.) (CRC Press/Taylor \& Francis, 2007).

40. Bell, M. A. \& Ball, M. J. Morphometric comparison of hippocampal microvasculature in ageing and demented people: diameters and densities. Acta Neuropathol. 53, 299-318 (1981).

41. Hunziker, O., Abdel'Al, S. \& Schulz, U. The aging human cerebral cortex: a stereological characterization of changes in the capillary net. J. Gerontol. $\mathbf{3 4}$ 345-350 (1979).

42. Kalaria, R. N. Cerebral vessels in ageing and Alzheimer's disease. Pharmacol. Ther. 72, 193-214 (1996).

43. Thirumangalakudi, L., Samany, P. G., Owoso, A., Wiskar, B. \& Grammas, P. Angiogenic proteins are expressed by brain blood vessels in Alzheimer's disease. J. Alzheimers Dis. 10, 111-118 (2006).
44. Fischer, V. W., Siddiqi, A. \& Yusufaly, Y. Altered angioarchitecture in selected areas of brains with Alzheimer's disease. Acta Neuropathol. 79, 672-679 (1990).

45. Rodrigue, K. M. et al. $\beta$-Amyloid burden in healthy aging. Neurology 78, 387-395 (2012)

46. Rodrigue, K. M., Kennedy, K. M. \& Park, D. C. Beta-amyloid deposition and the aging brain. Neuropsychol. Rev. 19, 436-450 (2009).

47. Nortley, R. et al. Amyloid $\beta$ oligomers constrict human capillaries in Alzheimer's disease via signaling to pericytes. Science $\mathbf{3 6 5}$, eaav9518 (2019).

48. Biron, K. E., Dickstein, D. L., Gopaul, R., Fenninger, F. \& Jefferies, W. A. Cessation of neoangiogenesis in Alzheimer's disease follows amyloid-beta immunization. Sci. Rep. 3, 1354 (2013).

49. Cameron, D. J. et al. Alzheimer's-related peptide amyloid- $\beta$ plays a conserved role in angiogenesis. PLOS ONE 7, e39598 (2012).

50. Cunvong, K, Huffmire, D., Ethell, D. W. \& Cameron, D. J. Amyloid- $\beta$ increases capillary bed density in the adult zebrafish retina. Invest. Ophthalmol. Vis. Sci. 54, 1516-1521 (2013)

51. Moore, S. et al. APP metabolism regulates tau proteostasis in human cerebral cortex neurons. Cell Rep. 11, 689-696 (2015).

52. Walsh, D. M., Klyubin, I., Fadeeva, J. V., Rowan, M. J. \& Selkoe, D. J. Amyloid-beta oligomers: their production, toxicity and therapeutic inhibition. Biochem. Soc. Trans. 30, 552-7 (2002).

53. Willem, M. et al. n-Secretase processing of APP inhibits neuronal activity in the hippocampus. Nature 526, 443-447 (2015).

54. Kofler, N. M., Cuervo, H., Uh, M. K., Murtomäki, A. \& Kitajewski, J. Combined deficiency of Notch1 and Notch3 causes pericyte dysfunction, models CADASIL and results in arteriovenous malformations. Sci. Rep. 5, 16449 (2015).

55. Lin, S. et al. Non-canonical NOTCH3 signalling limits tumour angiogenesis. Nat. Commun. 8, 16074 (2017).

56. Zimrin, A. B. et al. An antisense oligonucleotide to the notch ligand jagged enhances fibroblast growth factor-induced angiogenesis in vitro. J. Biol. Chem. 271, 32499-32502 (1996).

57. Groot, A. J. et al. Regulated proteolysis of NOTCH2 and NOTCH3 receptors by ADAM10 and presenilins. Mol. Cell. Biol. 34, 2822-2832 (2014).

58. Liu, H., Kennard, S. \& Lilly, B. NOTCH3 expression is induced in mural cells through an autoregulatory loop that requires endothelial-expressed JAGGED1. Circ. Res. 104, 466-475 (2009).

59. Konishi, J. et al. Gamma-secretase inhibitor prevents Notch3 activation and reduces proliferation in human lung cancers. Cancer Res. 67, 8051-8057 (2007).

60. Kalén, M. et al. Gamma-secretase inhibitor treatment promotes VEGF-A-driven blood vessel growth and vascular leakage but disrupts neovascular perfusion. PLOS ONE 6, e18709 (2011).

61. Zhang, Y. \& Xu, H. Substrate check of $Y$-secretase. Nat. Struct. Mol. Biol. 17, 140-141 (2010).

62. Berezovska, O. et al. Notch1 and amyloid precursor protein are competitive substrates for presenilin1-dependent $\gamma$-secretase cleavage. J. Biol. Chem. 276, 30018-30023 (2001)

63. Winkler, E. A., Sagare, A. P. \& Zlokovic, B. V. The pericyte: a forgotten cell type with important implications for Alzheimer's disease? Brain Pathol. 24, 371-386 (2014).

64. Ribatti, D., Nico, B. \& Crivellato, E. The role of pericytes in angiogenesis. Int. J. Dev. Biol. 55, 261-268 (2011)

65. Darland, D. C. et al. Pericyte production of cell-associated VEGF is differentiation-dependent and is associated with endothelial survival. Dev. Biol. 264, 275-288 (2003)

66. Ozerdem, U. \& Stallcup, W. B. Pathological angiogenesis is reduced by targeting pericytes via the NG2 proteoglycan. Angiogenesis 7, 269-276 (2004).

67. Simonavicius, $\mathrm{N}$. et al. Pericytes promote selective vessel regression to regulate vascular patterning. Blood 120, 1516-1527 (2012).

68. Bergers, G. \& Song, S. The role of pericytes in blood-vessel formation and maintenance. Neuro-Oncol. 7, 452-464 (2005).

69. Mcllroy, M., O'Rourke, M., McKeown, S. R., Hirst, D. G. \& Robson, T. Pericytes influence endothelial cell growth characteristics: Role of plasminogen activator inhibitor type 1 (PAI-1). Cardiovasc. Res. 69, 207-217 (2006).

70. Paris, D. et al. Inhibition of angiogenesis and tumor growth by $\beta$ and $\gamma$ secretase inhibitors. Eur. J. Pharmacol. 514, 1-15 (2005).

71. Cai, J. et al. $\beta$-Secretase (BACE1) inhibition causes retinal pathology by vascular dysregulation and accumulation of age pigment. EMBO Mol. Med. 4, 980-991 (2012). 
72. Luna, S., Cameron, D. J. \& Ethell, D. W. Amyloid- $\beta$ and APP deficiencies cause severe cerebrovascular defects: important work for an old villain. PLOS ONE 8 , e75052 (2013).

73. Hu, X., He, W., Luo, X., Tsubota, K. E. \& Yan, R. BACE1 regulates hippocampal astrogenesis via the Jagged1-Notch pathway. Cell Rep. 4, 40-49 (2013).

74. Oakley, H. et al. Intraneuronal $\beta$-amyloid aggregates, neurodegeneration, and neuron loss in transgenic mice with five familial Alzheimer's disease mutations: potential factors in amyloid plaque formation. J. Neurosci. 26, 10129-10140 (2006).

75. De Simoni, A., MY \& Yu, L. Preparation of organotypic hippocampal slice cultures: interface method. Nat. Protoc. 1, 1439-1445 (2006).

76. Stine, W. B., Jungbauer, L., Yu, C. \& LaDu, M. J. Preparing synthetic $\mathrm{A} \beta$ in different aggregation states. Methods Mol. Biol. 670, 13-32 (2011). 